\title{
97. Resistance to Ethanol Vapour of the Eggs and Larvae of Silkworm.
}

\author{
By Zyuiti Kuwana.
}

Imperial Sericultural Experiment Station, Tokyo.

(Comm. by N. YATSU, M.I.A., Oet. 12, 1940.)

In the hope of finding a key for the analysis of the physiological change of insects with the developmental stage, especially with ecdysis, experiments were carried out concerning the resistance to ethanol vapour of the eggs and larvae of silkworm.

\section{The reistance of eggs.}

The silkworm-eggs were exposed to ethanol vapour at $25^{\circ} \mathrm{C}$ for varying lengths of time from 30 minutes to 24 hours at various stages from 1 hour after the oviposition to immediately before hatching. Experimental procedure was as follows. Cotton soaked with absolute ethanol was . placed on the bottom of a Scheibler's desiccator about 5 litres in capacity. Eggs were introduced through a cock at the side of the desiccater. The eggs thus treated were kept afterward at $25^{\circ} \mathrm{C}$ and examined for the rate of hatching. About 100 eggs were used for each experiment that was repeated three times. Materials used were non-hibernant eggs of the race, Daizō and SY, and hibernant eggs of the race, E. 18, od, and SY. Observation of the hibernant eggs was limited to the post-diapause stage.

Non-hibernant eggs. Experiments were undertaken in 1 hour, 12 hours, and 18 hours after the oviposition respectively and afterward every 24 hours. Results are summarized in Fig. 1. Eggs treated with

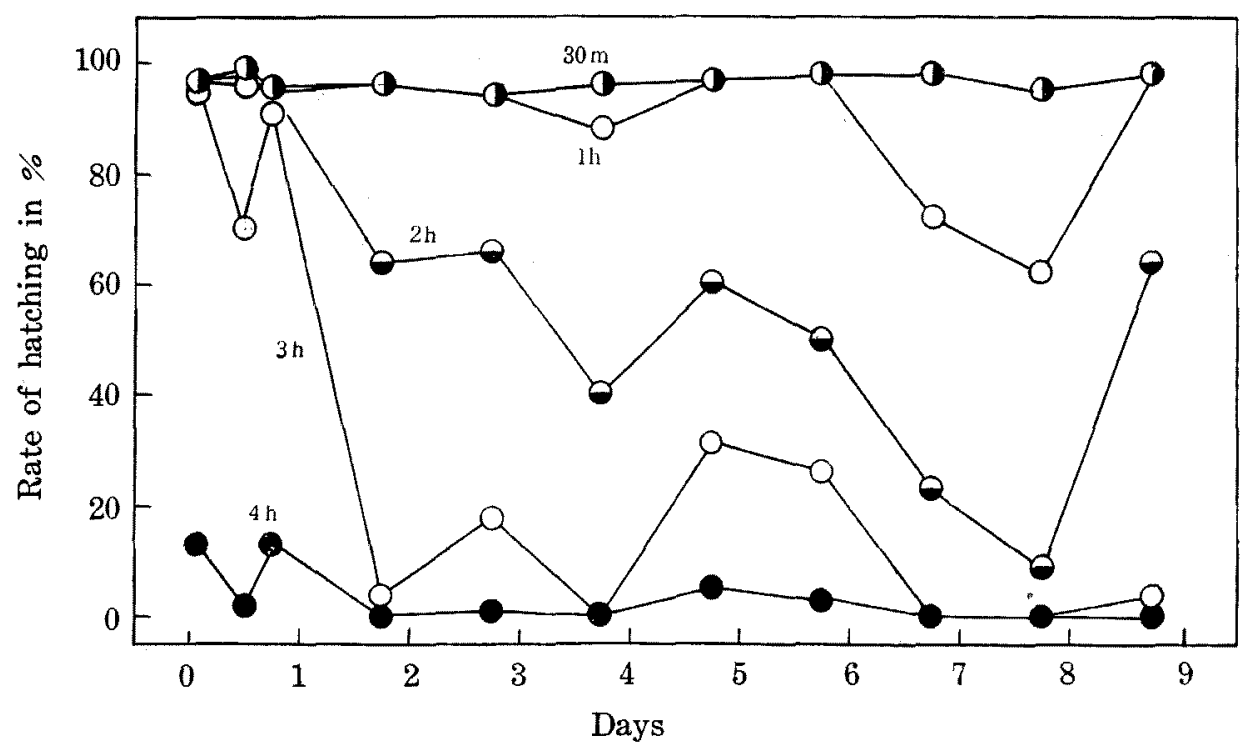

Fig. 1 Rate of hatching in per cent of the non-hibernant eggs treated from 30 minutes to 4 hours, plotted against age in days after oviposition. 
ethanol vapour within an hour after the oviposition for not longer than 3 hours, hatched quite normally with the rate of about 95 per cent; by the treatment longer than 4 hours the rate of hatching was much decreased. From the second day of incubation on, the eggs became less resistant. The least resistant period was in the penultimate day of the incubation, when even the treatment of an hour decreased the rate of hatching by about 40 per cent. Immediately before hatching, the rate slightly increased. At two or three days after oviposition, an orange colour appeared in the serosa the next day of 5 hour treatment. It is interesting to note that the same colour appears in exposing the eggs to $\mathrm{CO}_{2}{ }^{1}$ )

Hibernant eggs. All the eggs employed were laid in July of the previous year, and kept from early February in a refrigerator of a temperature of $2.5^{\circ} \mathrm{C}$. They were taken out of the refrigerator in April for the present experiment. Eggs treated with the ethanol

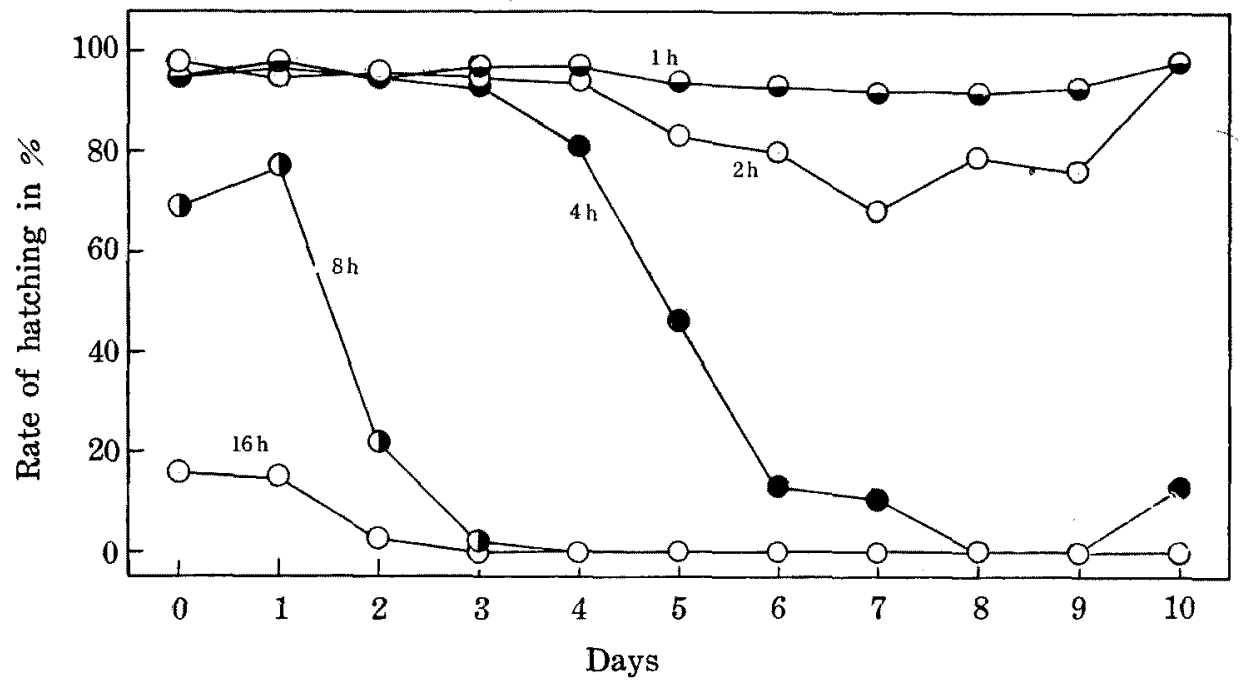

Fig. 2 Rate of hatching in per cent of the hibernant eggs treated from 1 hour to 16 hours, plotted against age in days after being taken out of refrigerator.

vapour in 30 minutes after being taken out from a refrigerator, for not longer than 4 hours seemed to be scarcely affected, the rate of hatching being about 95 per cent. By the treatment longer than 16 hours, the rate decreased to nearly 0 per cent. From the third day of the incubation on, the eggs became less resistant. As in the nonhibernant eggs, the least resistant period seems to be 1 or 2 days before hatching.

In both the hibernant and the non-hibernant cases, all the eggs treated not longer than 4 hours, as a whole, were able to arrive at nearly the last embryonal stage, where those seriously affected died prior to hatching. The eggs which succeeded to hatch out developed quite normally. 
The resistance of larvae.

Observations were undertaken from the second day of the first larval instar to the ripening period of the fifth larval instar. The materials used were E. 18, N. 107, SY and Daizō. Five larvae were introduced at a time into the desiccator, and the time for becoming fully inactive to a mechanical stimulus was measured for each larva. The stimulus given was gentle shaking of the vessel. From this inactive state the larvae sometimes recovered, and sometimes not. The resistance of the larvae was measured in terms of the time-to-anaesthesia, since no better criterion has yet been found to determine whether or not the larvae die completely. Ten observations were repeated for each experiment at $20^{\circ}, 25^{\circ}$ and $30^{\circ} \mathrm{C}$; at temperatures lower than $20^{\circ}$, the larvae were so inactive that the observation of the reaction to the external stimulus was difficult. The range of deviation of the time-toanaesthesia was \pm 20 minutes at most.

The results of the experiments are summarized in Fig. 3. First the change in the resistance during one larval instar will be examined. Starting from the beginning of an instar, the resistance increases as worms develop, especially on entering the sleeping period. After ecdysis, however, they become less resistant, the time-to-anaesthesia being, as a whole, even shorter than that of the later half of the previous instar. For example, in the first day of the fourth instar, the averaged timeto-anaesthesia is 38 minutes at $25^{\circ} \mathrm{C}$; in the third day, 70 minutes; in the fourth sleep, 97 minutes, and in the first day of the fifth instar,

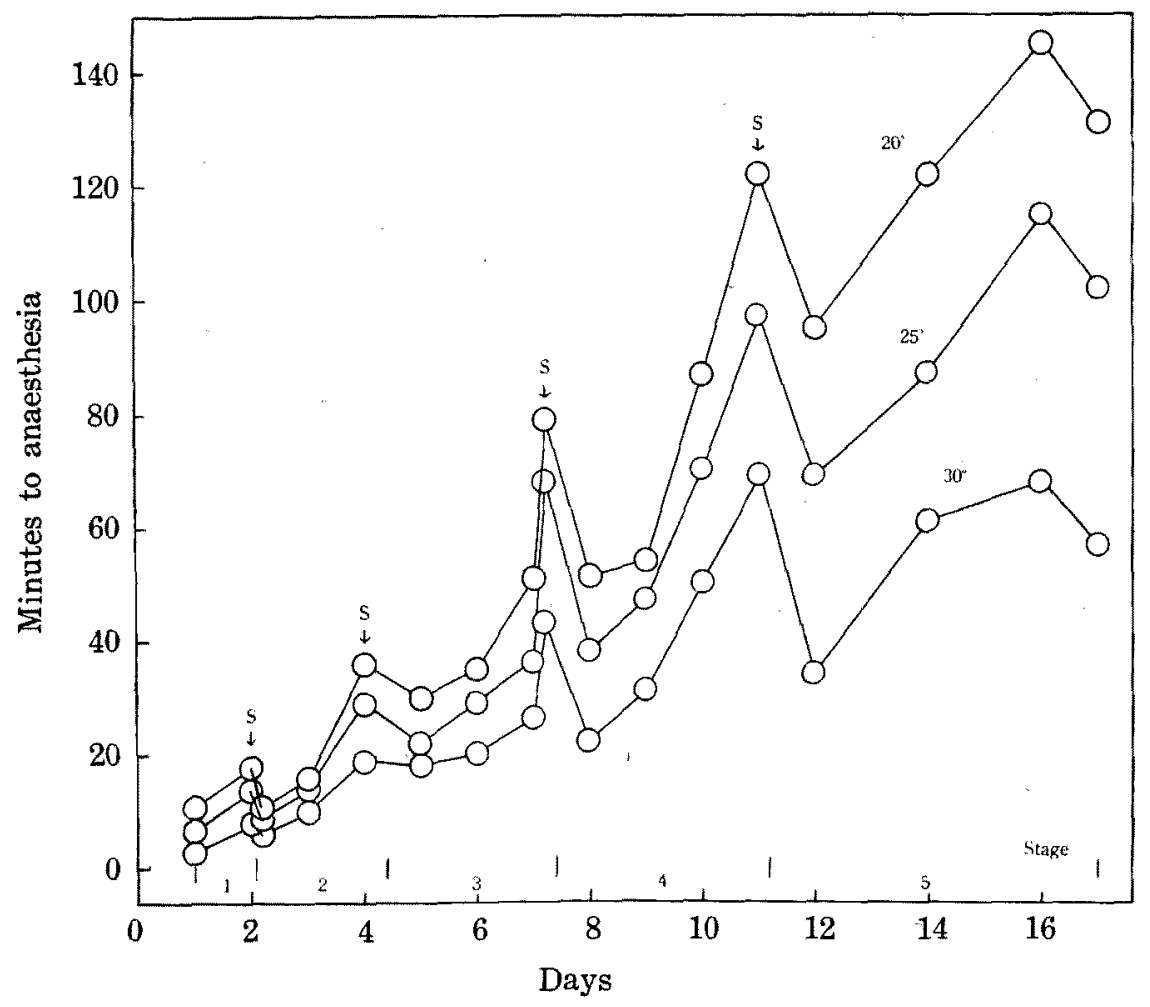

Fig. 3 Time-to-anaesthesia of larvae in minutes plotted against age in days after hatching. " $\mathrm{S}$ " represents sleeping period. 


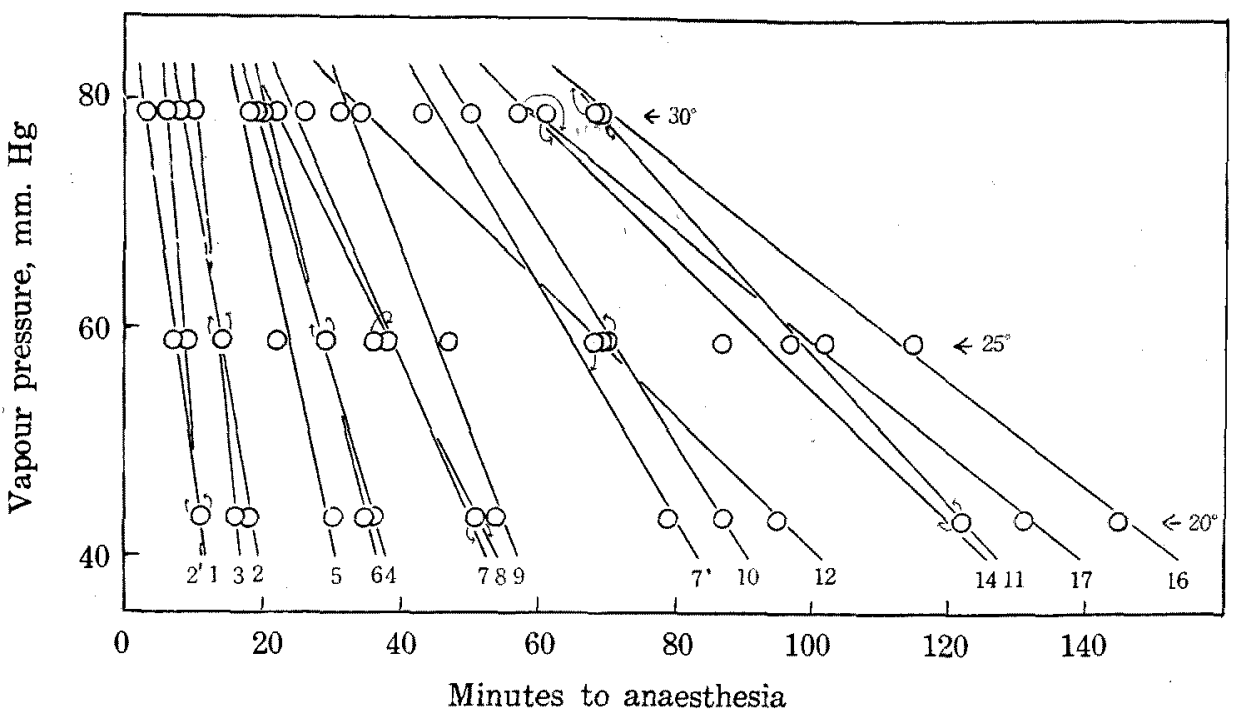

Fig. 4 Time-to-anaesthesia of larvae in minutes plotted against vapour pressure of ethanol in $\mathrm{mm}$. Hg. Numbers representing age in days after hatching.

69 minutes. On entering the ripening period the time decreases slightly.

On examining the whole course of the larval stage, it may be pointed out that the time-to-anaesthesia changes, from an instar to the next, following a succession of convexed curves, each of which corresponds to an instar. But, if only a certain corresponding period of each larval instar, for instance the sleep period, is considered, it will be seen that the time-to-anaesthesia is, in general, a rectilinear function of age.

Next the temperature relation of the resistance will be examined. It was observed with Drosophila ${ }^{\text {2) }}$ that the time-to-death ${ }^{2)}$ is a rectilinear function of the vapour pressure of ethanol and that the temperature has almost no significance but to regulate the vapour pressure. This fact seems to apply also to the silkworm larvae. When the fourth instar larvae of Daizō were placed in the vessel, in which were dropped $0.15 \mathrm{ml}$ ethanol (a quantity not sufficient for saturation of vapour), the average time-to-anaesthesia was the same, about 90 minutes both at $20^{\circ}$ and at $30^{\circ} \mathrm{C}$. On the other hand the larvae of the same lot given $0.3 \mathrm{ml}$ became inactive in 60 minutes. The vapour pressure ${ }^{3)}$ relation of the time-to-anaesthesia of the silkworm-larvae is shown in Fig. 4. The slope decreases with the development, showing the least value of an instar at the sleep period. On entering the following instar, the slope becomes steeper again, and continues to decrease until the sleep. This manner of change continues successively from an instar to the next.

Conclusion. 1) On examining the post-diapause period, the hibernant eggs seem to be more resistant to the ethanol vapour than the

1) Crozier, W. J., G. Pineus and P. A. Zahl 1936 J. Gen. Physiol., 19, 523.

2) The death point of the adult Drosophila is easily determined by the drawingin of the legs.

3) Data from the Landolt-Börnstein's Physikalisch-chemische Tabellen, 1923, Berlin. 
non-hibernant eggs. The first day of the embryonal development appears to be most resistant.") The least resistant point is found in nearly the last day of the embryonal development, while in the day of hatching the resistance increases to a considerable degree. The embryos immediately before hatching seem to be more resistant than the newly hatched larvae.

2) The time-to-anaesthesia of the larvae continues to increase in general with growth, though a periodic decrease of the resistance is found after each ecdysis. The maximal resistance of each instar at the sleeping period seems to be in close relation to the low metabolic rate in this period. ${ }^{2}$

1) By the treatment with $\mathrm{HCl}$, or an abnormally high temperature (Yamaguti, T. 1939 J. Sericul. Sci., 10, 208) or centrifugation (Matsumura, S. 1939 Bull. Sericul. and Silk-Ind., 11, 164), a period of a minimal resistance is found in 1-2 hours and 12 hours after ovi-position. It seems probable that the effect of ethanol vapour on the silkworm-eggs is not of the same kind as that of $\mathrm{HCl}$, a high temperature and centrifugation. The resistance of the eggs of Chilo simplex to nicotine decreases with age, as in the present result (Misaka, K. 1932 Bull. Imp. Agr. Exp. Sta., 3, 225; 1938 J. Imp. Agr. Exp. Sta., 3, 239).

2) Itaya, K. 1940 J. Sericul. Sci, 11, 113. 